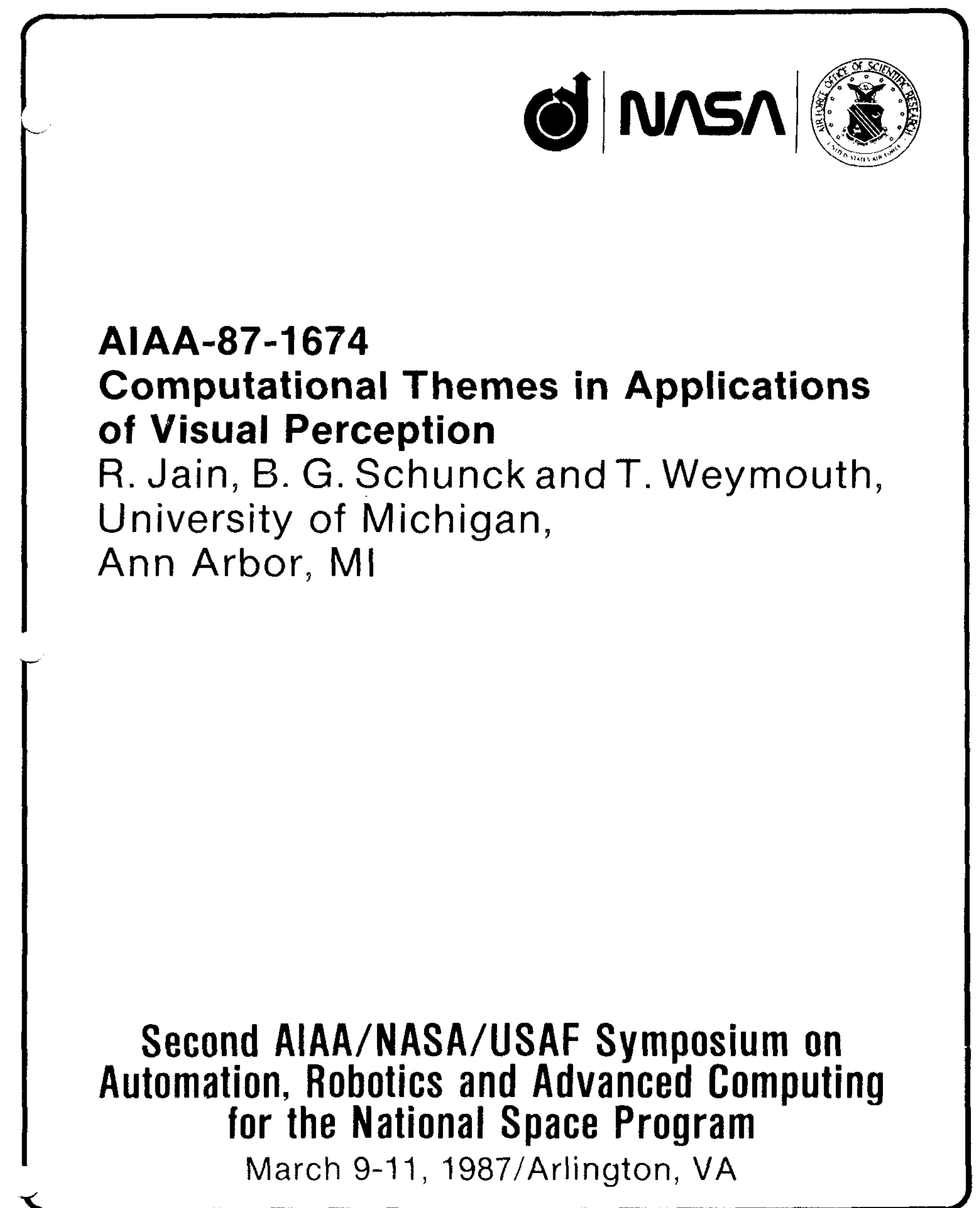

For permission to copy or republish. contact the American Institute of Aeronautics and Astronautics 1633 Broadway. New York. NY 10019 


\title{
Computational Themes in Applications of Visual Perception
}

\author{
Ramesh Jain \\ Brian G. Schunck \\ Terry Weymouth \\ Computer Vision Research Laboratory \\ Department of Electrical Engineering and Computer Science \\ University of Michigan \\ Ann Arbor, MI 48109-2122
}

\begin{abstract}
The paper surnmarizes the current research in the Computer Vision Research Laboratory at the University of Michigan. The laboratory concentrates on developing generic vision algorithms for industrial applications. Generic vision algorithms can be applied to a wide variety of inspection problems. The paper includes a discussion of the current state of the machine vision industry and provides recommendations for improving the transfer of vision technology from research to practice.
\end{abstract}

\section{Introduction}

The University of Michigan recently formed a laboratory, called the Computer Vision Research Laboratory, within the Department of Electrical Engineering and Computer Science for research in computer vision. The Computer Vision Research Laboratory will evolve through interaction among the researchers of various related disciplines and through inductrial and government contacts.

The research program in the Computer Vision Laboratory concentrates on the development of generic vision algorithms that can be applied to a variety of situations. Generic perception algorithms are simple, reliable modules that hide the details of perception in a packaged hardware or software component that can be used by applications specialists who are not themselves expert vision researchers. Generic vision algorithms are not developed for specific applications, but are designed to solve a vision task that is part of many different inspection problems that occur in different applications in different industries. In developing generic vision algorithms, researchers leverage their efforts by providing solutions to classes of vision tasks while removing the details of the application from the research efforts. This insulates the vision researchers from the details of the application and allows them to concentrate on understanding the vision problems.

Another aspect of research on generic vision algorithms is that the algorithms are classified according to properties that are meaningful in the context of the emerging knowledge of vision fundamentals, as opposed to classification according to applications areas. There are no automotive vision algorithms or aerospace vision algorithms; vision algorithms are not specific to any one industry. Vision algorithms are defined by the properties of the vision processing task; that is, in terms that depend on the vision processing itself, rather than on some intended use for the algorithm.

The nature of the problems of applying vision to specific applications has not been widely perceived. The current macroeconomic system of machine vision is inappropriate. The solution is to place machine vision technology in the hands of the end users who are familiar with the requirements of their environment. Vision technology should be like any other instrumentation: plug it in and use it to make measurements. It is not practical for the developers of machine vision applications to perform the development specific to the application and to develop the "instrumentation" required for the development at the same time. To change the current, untenable system, vision researchers must pro duce generic vision algorithms that can be used by vision systems developers in a variety of applications.

Researchers are currently addressing problems related to vision engineering but without much, if any, concern for designing machine vision systems. The focus of the Computer Vision Research Laboratory on vision algorithms that are generic, rather than applications specific, meets needs not addressed by other research institutions. The Computer Vision Research Laboratory provides a focus for the synergistic interaction of researchers aided by interactions with industry. The goal of research in the laboratory is to address fundamental problems blocking advances in the design of machine vision systems for various applications. The research addresses basic issues in computational vision research and the design of systems that can perform vision tasks in real time for a given application. New engineering techniques for designing systems are being developed that can perform tasks in an unstructured environment. The research may also advance the understanding of biological vision systems. 


\section{Research Problems}

Visual perception is the process of interpreting measurements such as light intensity and range that relate to the projection of surfaces in a scene onto the image plane. The central problem in vision is the reconstruction of surface structure and properties from the projection onto the image plane. This paper outlines some of the research problems currently being explored in the Computer Vision Rescarch I,aboratory.

- Dynarnic vision

- Range images

- Knowledge-based perception systems

- Computer architectures for vision algorithms

- Sensor integration

- Bridging the gap between research results and applications

\section{Dynamic Vision}

Motion provides valuable clues to surface structure. Algorithrns for interpreting sequences of images can provide measurements that are very useful for applications. Image flow research has many applications including passive sensing systems for autonomous mobility, machine inspection of surface structure, passive sensors for aircraft and satellite docking systems, and image compression.

\subsection{Background}

The last few years have seen increasing intcrest in dynamic scene analysis. The input to a dynamic scene analysis system is a sequence of images. A major problem in a computer vision system is to recover the information about objects in a scene from images. This problem cannot be solved without some assumptions about the world. A sequence of frames provides the additional dimension of time for recovering the information about a 3-dimensional world that is lost in the projection process. Multiple views of a moving object acquired using a stationary camera may allow recovery of the structure of the object [45]. A mobile camera may be used to acquire information about the structure of the stationary objects in a scene using optical flow $[11\}$, axial motion stereo $(25,32]$, and other methods $[21]$.

Many researchers in the psychology of vision support the recovery of information from image sequences, rather than from a single image $(11,31\}$. Gibson $[11]$ argued in support of active information pick-up by the observer in an environment. Neisser [31] proposed a model in which the perceptual processes continually interact with the incoming information to verify expectations formed on the basis of available information. In computer vision systems, the power of exploiting motion, even with such noise sensitive approaches as difference pictures and accumulated difference pictures, has been demonstrated on complex, real world scenes $[20 \mid$. Many researchers are addressing the problem of recovering information in dynamic scenes; but due to the legary of static scenes, most researchers are approaching the recovery problem using just two or three frames of a sequence. This restricts the results to quasi-dynamic scene analysis, rather than true dynamic scene analysis. The information recovery process requires constraints about the scene. The analysis based on small numbers of frames rests on assumptions that ignore the most important information in dynamic scenes.

The viewpoint of the Computer Vision Rescarch Laboratory is that image understanding is a dynamic process. Dvnamic vision algorithms cope with the error-filled visual world by exploiting redundant information in the inage sequence. Current research efforts are developing a qualitative approach to vision that uses only relative information available in a sequence to infer relationships between objects in a scene.

\subsection{Segmentation}

In many dynamic scene analysis systems, the goal is to recognize moving objects and to find their motion characteristics. If the scene is acquired using a stationary camera, then segmentation generally refers to the separation of moving components from stationary components in the scene and identification of individual moving objects based on velocity or some other characteristic. For the case of a moving camera, the segmentation task may be the same as above or may involve further segmentation of the stationary components of the scene by exploiting the motion of the carriera. Most research efforts for the segmentation of dynamic scenes have been concerned with the extraction of the images of the moving objects observed by a stationary camera. It has long been argued by researchers in perception $\{11,45\}$ that motion cues aid the segmentation process. Computer vision techniques for segmenting dynamic scenes perform well in comparison to those for the segmentation of stationary scenes. The segmentation of moving camera scenes into their stationary and nonstationary components has received attention only recently $\{22 \mid$. The major problem in the segmentation of moving observer scenes is that every surface in the scene shows motion in the image. For separation of moving object images, the motion component assigned to various surfaces in the images due to the motion of the camera must be removed. The fact that the image motion of a surface depends on its distance from the camera and the surface structure complicates the situation.

Jain developed techniques for the segmentation of dynamic scenes $(19,20,22\}$. These techniques have been used in many applications. Sandia Laboratories is developing systems for tracking objects for Army applications using techniques based on differencing using a likelihood ratio. Recently research led to a new approach for segmentation using accumulative difference pictures that may be implemented easily on special hardware [23].

\subsection{Image Flow}

Image flow is the velocity field in the image plane that arises due to the projection of moving patterns in the scene onto the image plane. The motion of patterns in the image plane 
Thay be due to the motion of the observer, the motion of objects in the scene, or both. The motion may also be ap. parent motion where change in the image betwen frames sives the illusion of motion [36].

The insent of this research is to discover new tnodels for image fow that will yield new algorithms for image flow estirration and analysis. Constraint equations that bether model the interactions between changes in object position

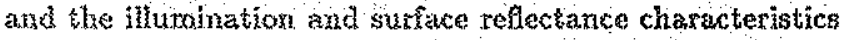
will naturally result in better algorithns and better undersçanding of existing algorichns.

prior image fow research developed an innage fow equa tion for smooth patterns of mage inadiance and smooth ve locity fields [27]. The equation was extended first to inage inadiance pattems with discontinuities and then to velocity fields with discontinutites $[55,56,60]$. Future research in inage flow constraine equations will aixn to increase our understanding of image fow characteristics. This will lead to new algorithrus for itoage fow estimation that incorporate the new continuity equations. Restrictions on the situations in which existing continutivy equations can be used will be discovered and these ingights will inaprove the performance of existing image fow estimation algorithms by pin pointing the situationg where the algorithras cannot be used.

\subsection{Motion stereo}

Deptl determination is a continuing problem in computer vision. Resenzch in this area is motivated by the need for target tracking, autonoraous veluicles concrol, visual prostheses for the blind, realistic fight trainers, and models of the human visual system. There is a plethora of depth determination techniques. Many diferent stereo systems for depth deterwination have been developed just in the last fow years.

Stereo infortantion can also be obtained using a aingle moving camera. Jain and $\mathrm{O}^{\prime} \mathrm{B}$-ien $[25,32]$ map images into a complex log space where the movement of the objects in two dimensions dute to the catnera rnotion becomes a translation along one axis in the new space. Given this phenomenon, the wotion comespondence problex is greatly reduced, since onfy a sanall strip of the new space needs to be zearched [18]. This constraint is similar to the epipolar constrents in atereo. In addition, the transform is scale and rotstion

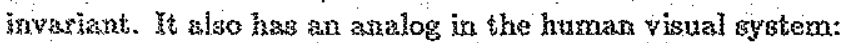
the mapping of the refinal space into the striate cortex is

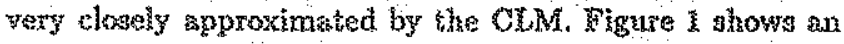
inarge and its mapping.

Optical How has bean stadied with the aira of recovering information about the environment and the motion of the observer. The egomotion complex logarithin mapping

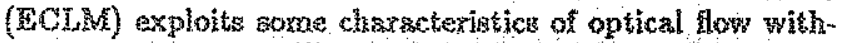
out computing it. The rapping combine acals, projection, axt rotation invariance of coxaplex los mapping with the chasscteristics of optical fow . This mapping is useful in segzyentsion of inage sequencs to recover images of moving objects. Fhe datance to stationary objexts can also be cora puted from the egomotion complex kogarithmic mapping. Using this mapping, rustion stereo and segraentation can be achieved in one ster. The feasibility of the approach has
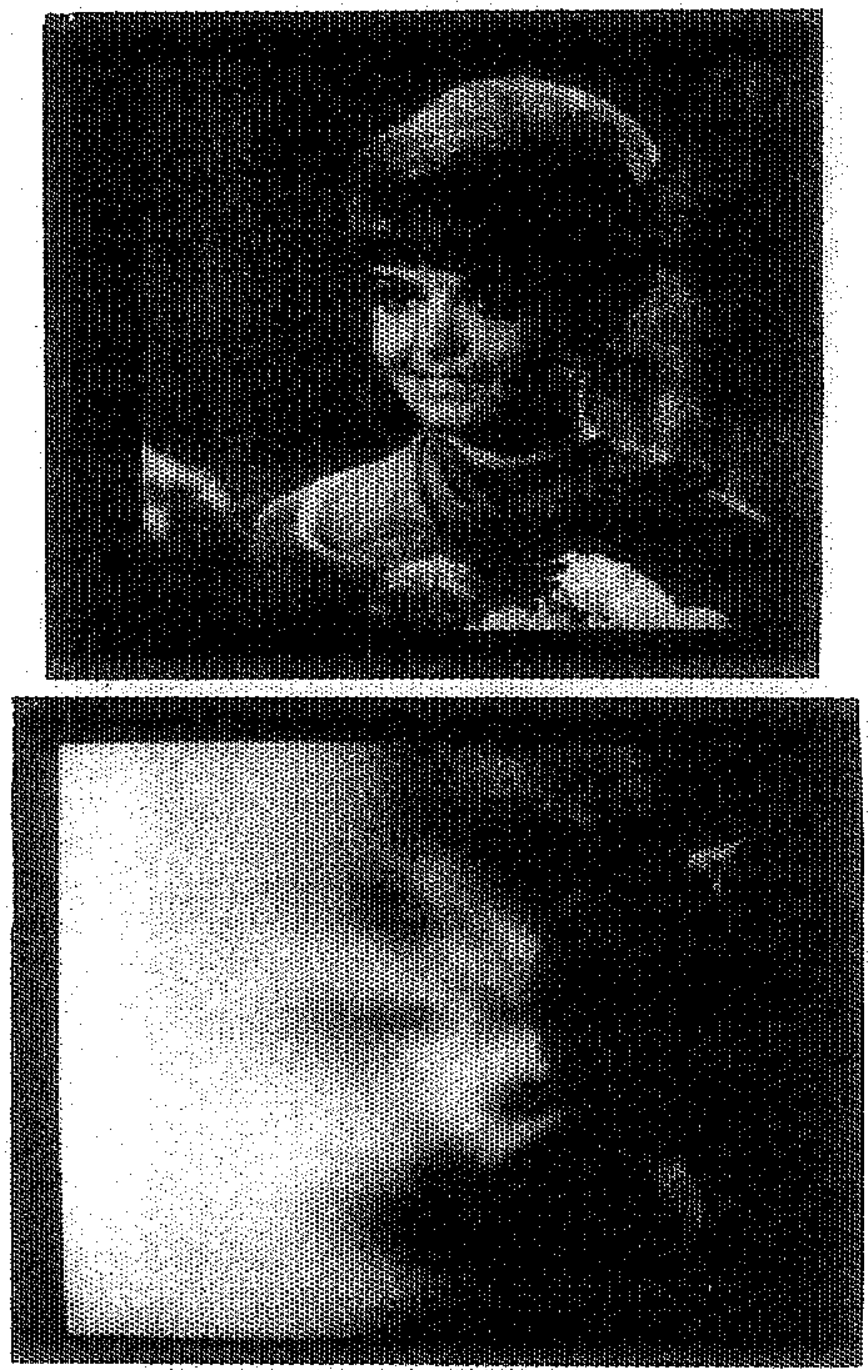

Figure it An image and its complex log mapping are shown in this figure. The mapping is similar to the retino-stxiate rrapping in primate visual systems. This mapping is very useful in segmentation and depth recovery in dynamic vision.

baen demonatated. The next step is to study ifs application in Aynamic scenes. This research should yield inoportant results for the navigation of wobile robots.

\section{Motron Tratectories}

Terative algorithms for etermining tratector of yoints in an extended frame sequence using goth pohertsee are being developed. The emphasis in this appronch is to exploit raso sion characteristics for establishing correspondence without aseuming rigidity of objecks. A grecty exclange skgorthtorn has been developed to implement of of applyiag this algorithm to a seçurence from the Sugermon xuryie are shown in Fis. 2. 

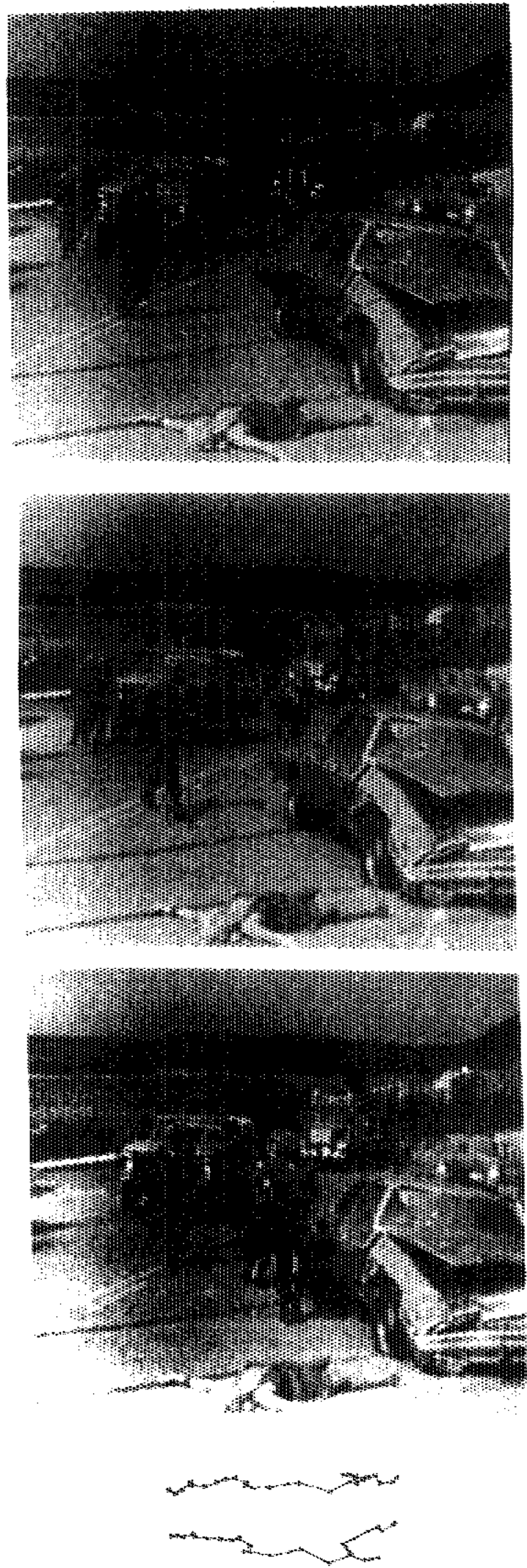

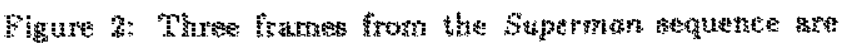

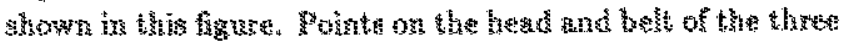

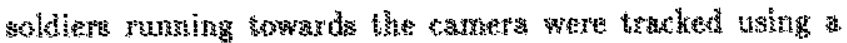

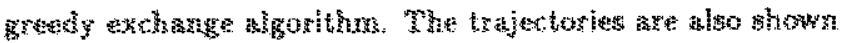
bext.

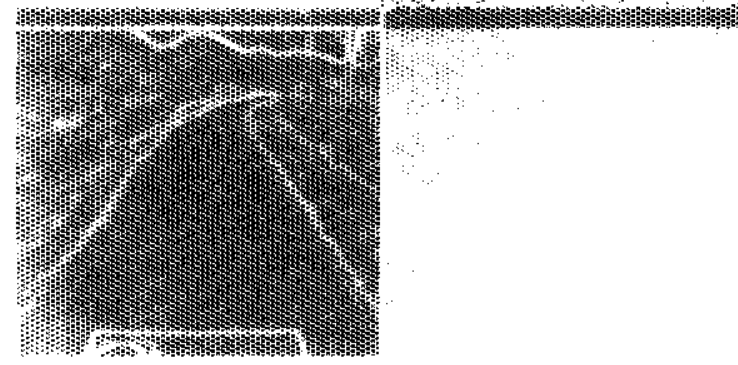

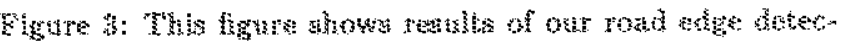

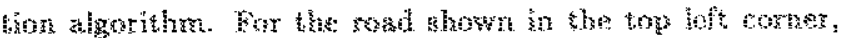

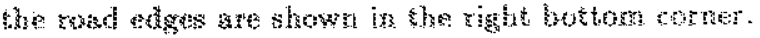

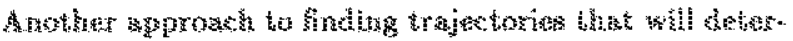

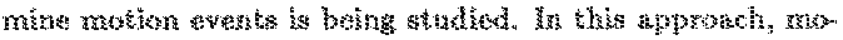

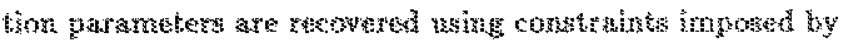

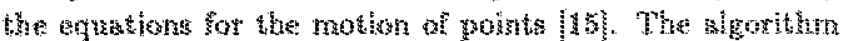

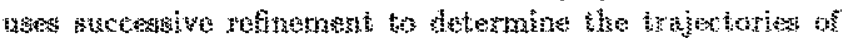

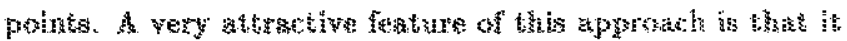

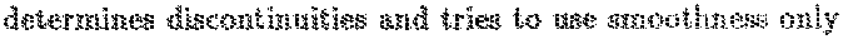

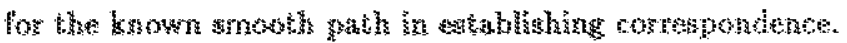

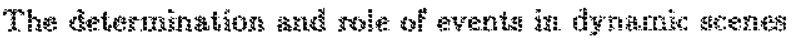

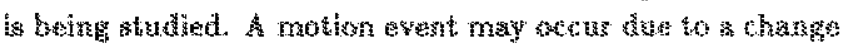

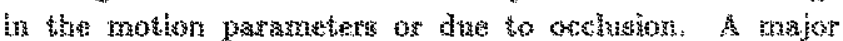

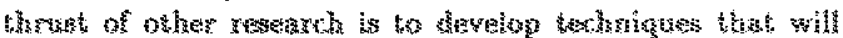

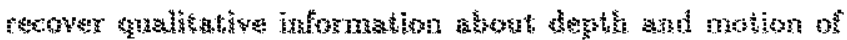

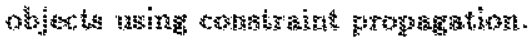

\section{Novigation}

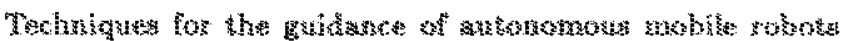

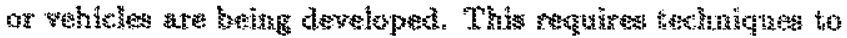

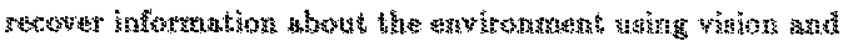

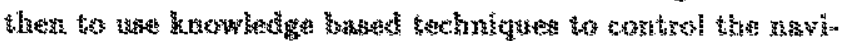

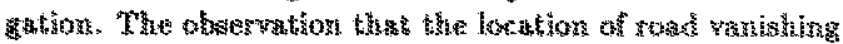

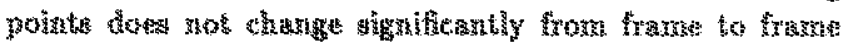

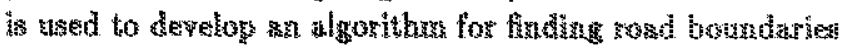

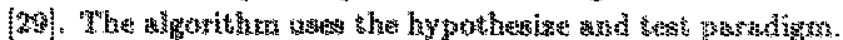

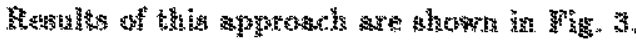




\section{Range Images}

The long terro goal of this groject is to develop technicues that will be useful in objest recognition and navigation using range information.

Tho last few years have seen increasing attention to the andysis of range images. Kange images may be obtained with passive methods, such as binocular stereo, or with range sensors. Thange inarges contain explicit information about suraces. This explict information facilitates recognition and location tasks in tany applications. The goal in range inage understaneling is to find robust symbolic surace descriptions that ase independent of viewpoint. Techniques so characterize surfaces in sange inages are being developed $\{5,4,24]$.

Suriaces are segmented uging local festures, such as Gaussian and mean curvatures and related differential geometric measures. The signs of the curvalures at each point in the irage are used to assign one of eight basic surface types to each point in the intage. "The next step is to develop techniques to idertity auface by grouping points using the surface type, spatial proximity, and other criteria. All grouping processes must conform to the sensed information since the dutixyate truthy in the sensed data. This stimulus bound approach uses sytabolic surface descriptions in the segmentration of ixyages. Figure shows a result of this segmentithon aprouch. The aymbolic surface descriptions will play important, role in many apulications.

\subsection{Recognition Methods}

Object recognition is a major motivation in most inargeunderstanding systems. Despite strenuous effors, only lim. ited success has been achieved.

The object recogntion task can be classified bascd on difficulty in several ways, One way is yased on the degree of uncervanty allowed in the object's position and orientation. This can range from no uncertainty, to uncertainty ony in its $2-\mathrm{D}$ position, shough uncertainty in both its $2-\mathrm{D}$ positon and rotation, wp to uncersanty in its 3-D position and orientation.

The izsk can be further classified based on the complexity of interactions allowed between objecss. In the simplesi case, each object to be recognimed must be completely vistble and surrounded by background. In a more complex case, objects are allowed to touch but not overlap. In the raost general case, objects are allowed to partially occhde one arother.

Most worl on the ochided objects problem has concentrated on the case where there is only one object type in the sicene. Agoriehme developed for this problem can be extended to cases of mattipla objerts types, simply by running the algorithu multiple times, once for each possible object type. This is nos deal, as it does not consider or take advantage of the gintaristes and differences among possible objess. Also the noconntion time increases linearly with the number of possible objexs, after a fixed time for pre processing. This can become o problem as the number of possible objocs typer increases.
A new mechod for object recognition called the feat tsre indexed hypotheses method is beirg developed. This method breaks the recognition process into two phases: hypotheses generation and hypothesis verification. By using features that occur multiple times in the possible object set, the number of features in the search can be greatly reduced generating a sxall number of false hypotheses that are easily rejected. By carefully selecting the features, the recognition time growth rate car be reduced to the square root of the nuxber of possible objects. This method also has the advantage that uniche features which are flifficult or impassible to find if the possible object set contains wany similar objects are not required. The method's tearibility is demonstrated by the resalts of a prototype two-dinemsional occludeci-parts recognition system.

\section{Krowledge-Based Perception Sys- terns}

Most complex tasks require spectakized knowledge, innage understanding is no excention. The last few years have secn an increasing application of knowledge in computer vision systems. The application of knowledge at different levels in a vision system is being studied.

\subsection{Krowledge-Based Algorithras}

An image interpretation system is a program that derives a scene description from a time-varying image. The ingut signal is a reciangular grid of signal saxxples taken at, regular time intervals $l(x, y, i)$. The sample can be taken in several frequency ranges (for example; red, green, and blue) and combined into a time-varying vector field $C(x, y, b)$. There is wide agreement that this characterimes the environmental data used by such a system $[9,17]$. On the other hand, it is tuuch more difficil to formulate a description of the outpus of an interpretation systera. Wn this project, the outyat of an interpretation system is a network representing the scene being viewed, an approach common to other interpretation systems. The nodes of such a network are groups of objects within the scene: individual objects, object, parts, or references to clusters of image events. Arcs in the network are labeled with relations between the objects. Since primitive object parks are uswally depicted as geometric solids or collections of joined suriace packles [10], these representations are included in the representation used in this work.

However, shere is no need to linit ourselves to only bhat type of representation. In sact, the sadidy of which types of primitives are tugeful and how they relate to the processing of the inage is part of our current research. The issues of which relations to use, how to vary thase relations with tirue, and how to theorporste the martakns jobject descriptions that occur ove sine are also beng studied.

The thage senuence is not the only sounce of data wsed by an interpretation systern. A large database of relational and descriptive incornation, ancluding information about processes and procedures, is also necessary tor image incer- 


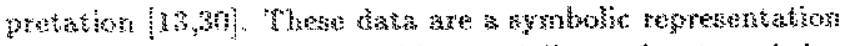

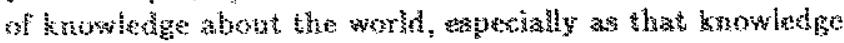
pertans to tho inderpretatios: problem; thus, the dathbase is wstally referred to as a knowledge base. For wathyle, if it is

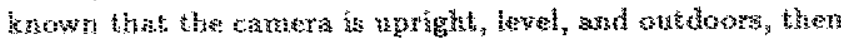

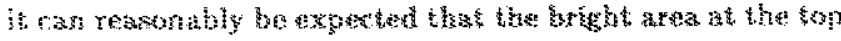

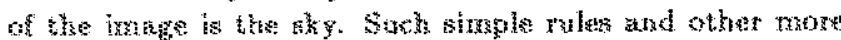

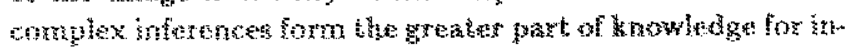

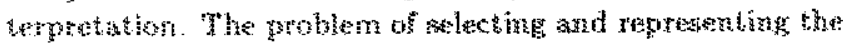

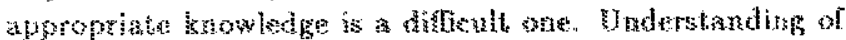

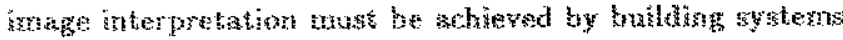
of processes that won in restricted tomatus. In sulding such system, the mites and proceses whin can swentably

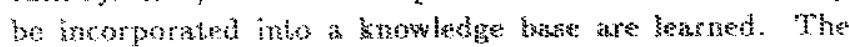

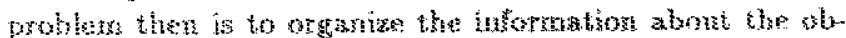
fext and the grocedures for using that informaton so thint

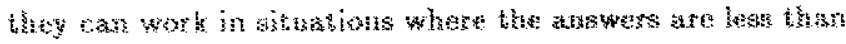
certsin.

The issuen whe basticaly these:

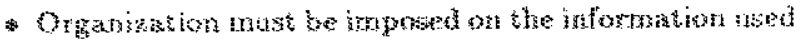
Fo image interpotation, becanse that information is

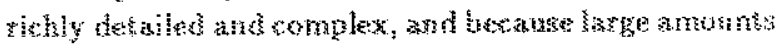
of informetom are seninged.

* Varied ypes of information (for example, relationsi,

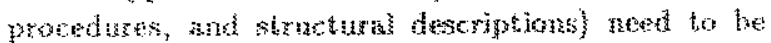

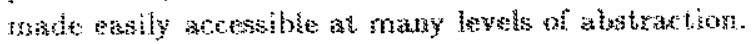

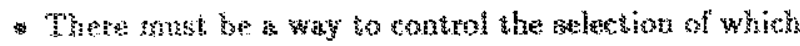
information is comsidered, which prostames arge att. vatek, and what information is pastest among prow frams.

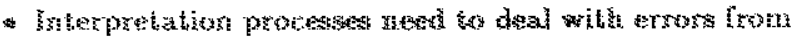

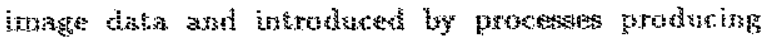
partibl intermetation.

* All components of the intergetetation system should be

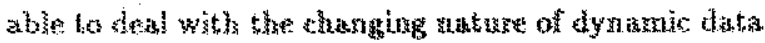
and the reorgatination of interyretationa that ocent when a proces creater an hypolsesen.

\section{Vision Computer Architecture}

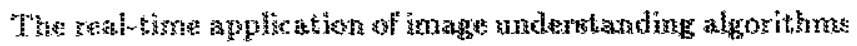
renuires complater hardware that ellows the algorithms bo be exeruted at bish mped. The

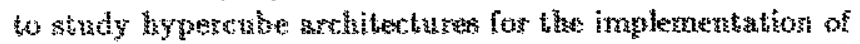
vision algoritaym.

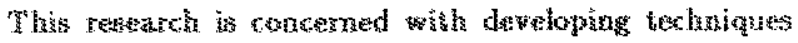

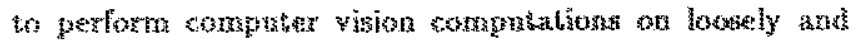

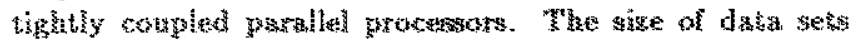

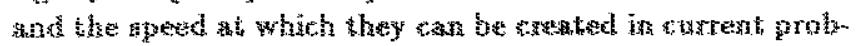

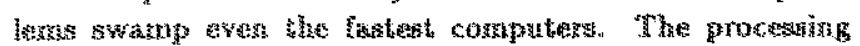

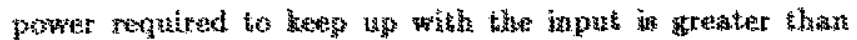

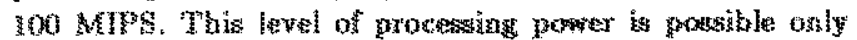

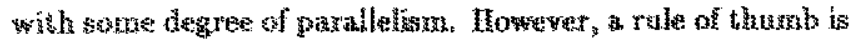
that yarallel procesors can rarely stustain an elferency level of greater than $20 \%$ [36]; thas the machune needs to bave

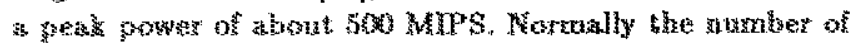

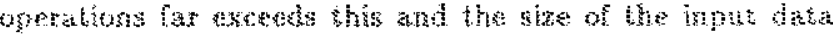

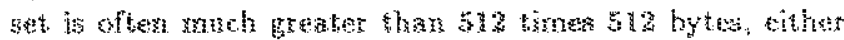

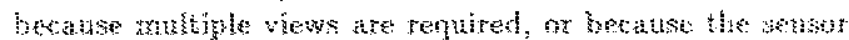

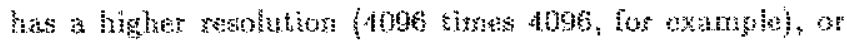
mere that one modality is its use.

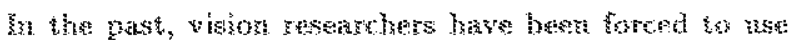

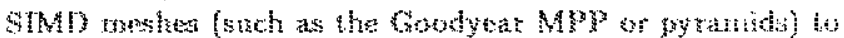

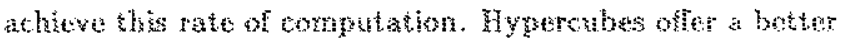

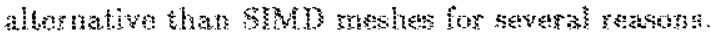

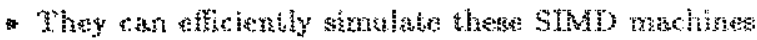

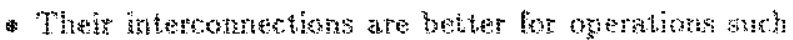

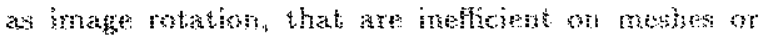
pyrantils

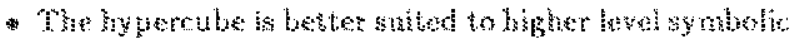

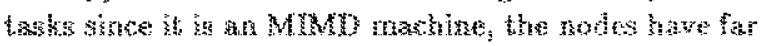

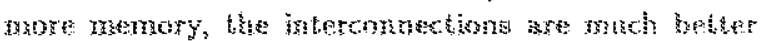
for the mortandam fromathen exchangt; and the

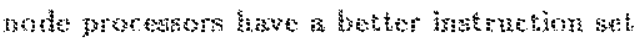

As an example of eflicinoy in visin tasks, a hypmone

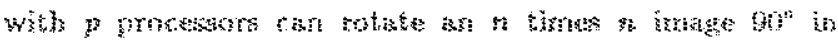

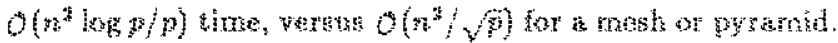

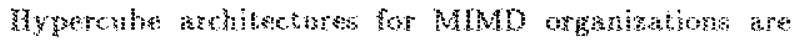

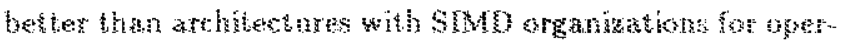

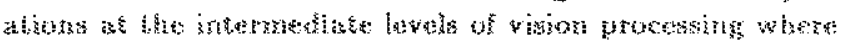

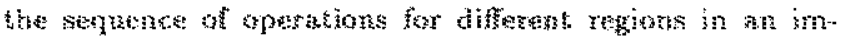

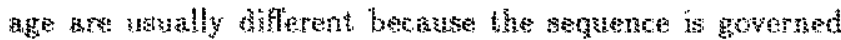
by properien of the region. For this type of frocestng, a

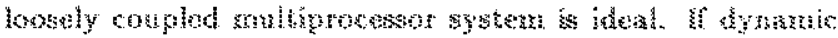

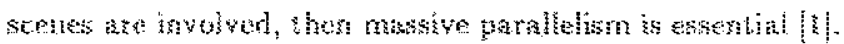

\section{Sensor Integration}

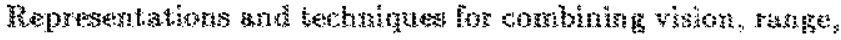

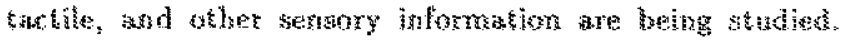
hepresentrations that will alow ensy infereske waing mult

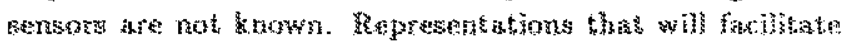

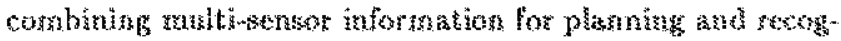

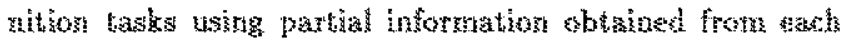

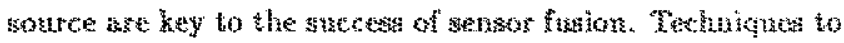

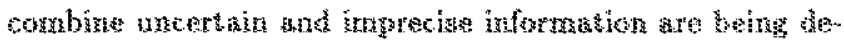

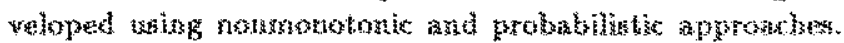

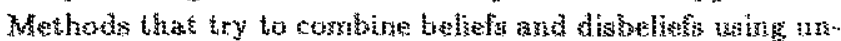
certanty calculus for solvang problezs in distritubst prob-

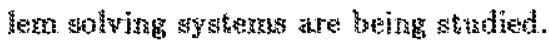

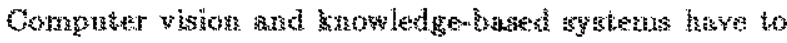

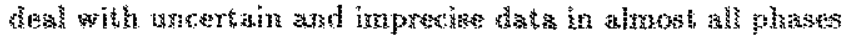

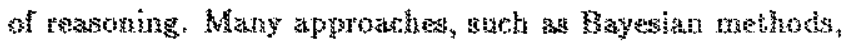

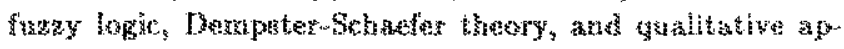

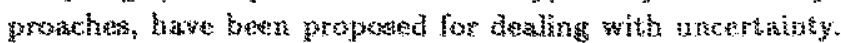

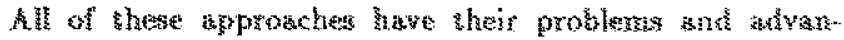

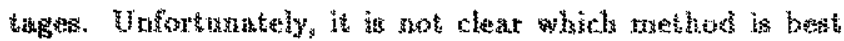

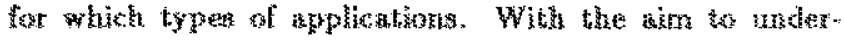

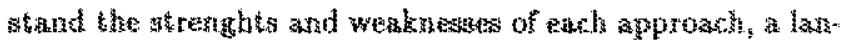

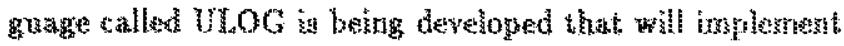

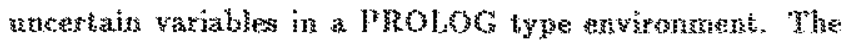

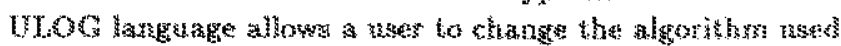




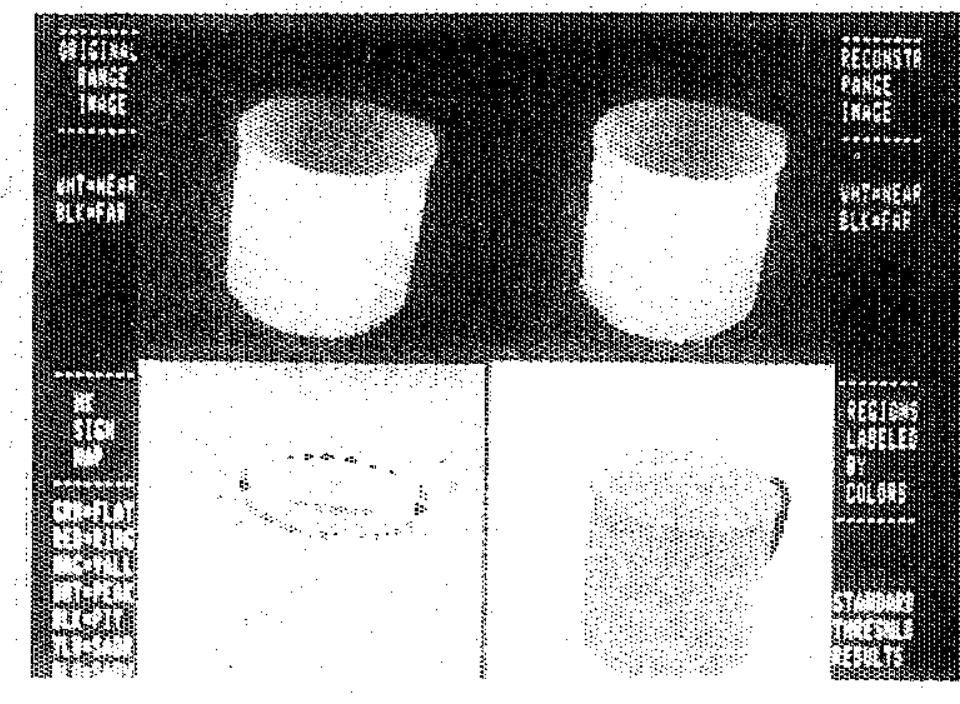

Figure A: A ronge image and its segmentation using Besl's and Jan's algorthro are shown in the top-lett and botfom-right conners respectively. Using the polynomial descriptors of the segmered surfaces, the originat image is reconstructed. This is shown at top-right.
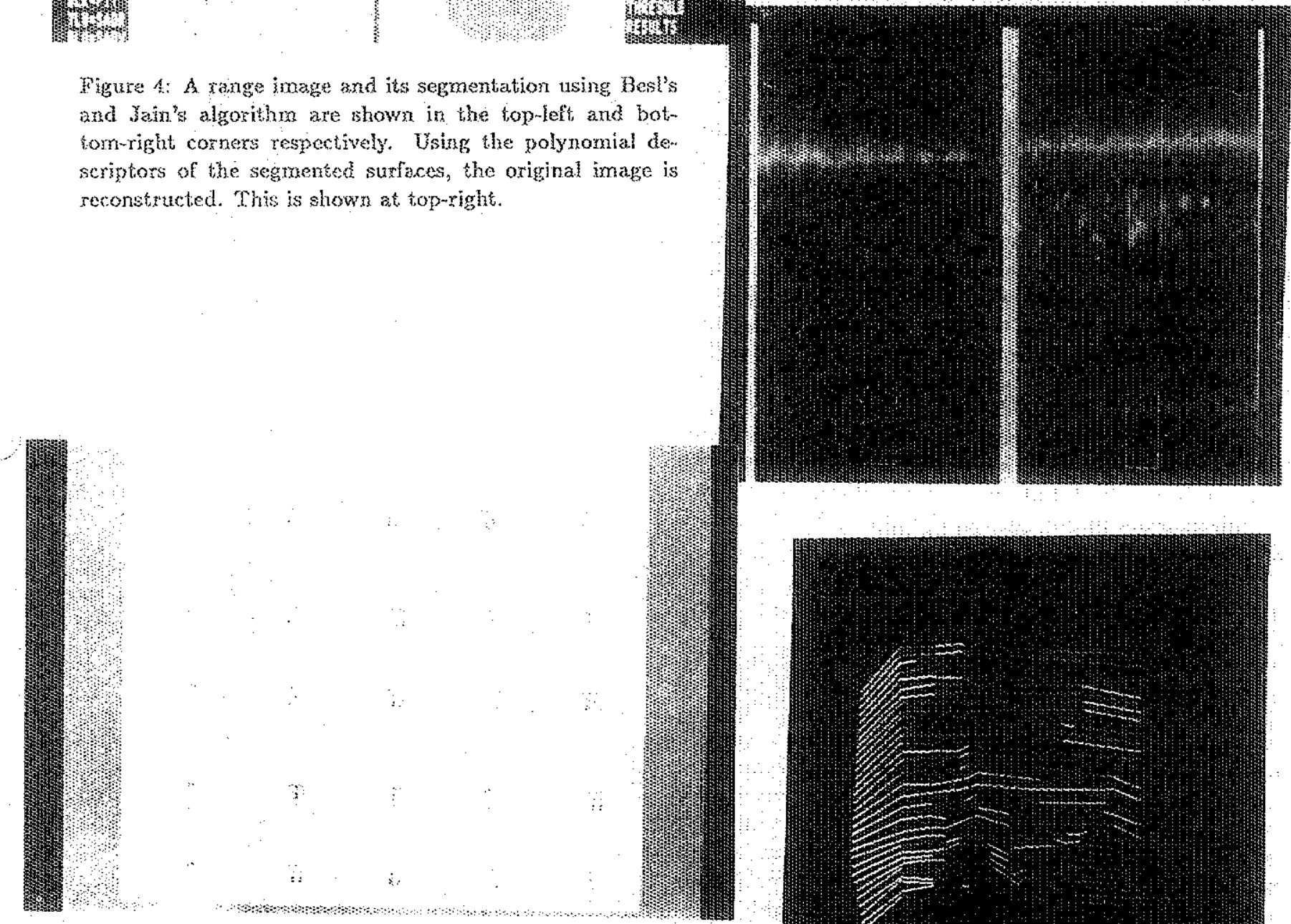

Figute 5: This figure shows an image showing the classification of solder joinss by our algorithm.

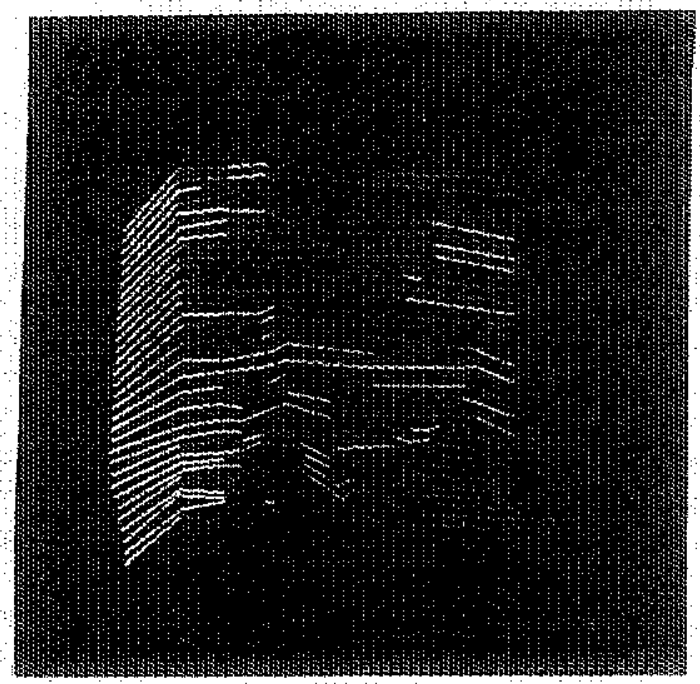

Figure 6: This figure shows an inage showing two SEM innages of a section of a wafer and the 3-D surface structure as reconstructed using our sem stereo algorithm. 


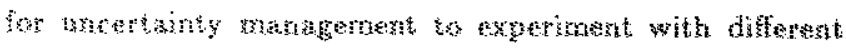
apporkm in orter bo hnd the best appond for a given poblent

\section{Applications}

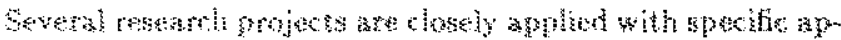

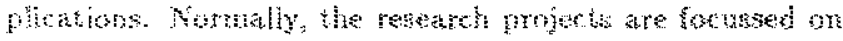

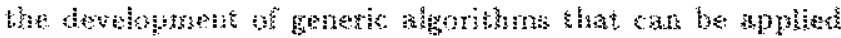

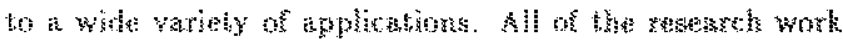

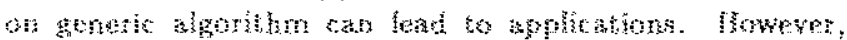

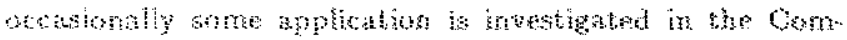

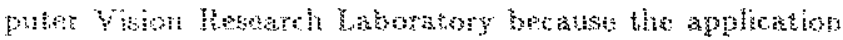

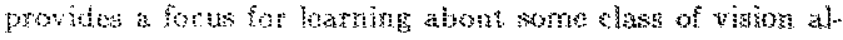

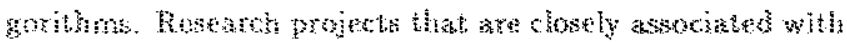

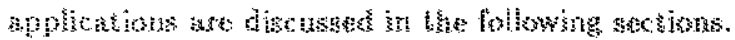

\subsection{Solder Joint Iraspection}

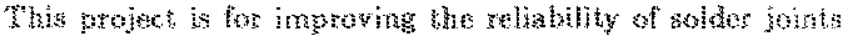

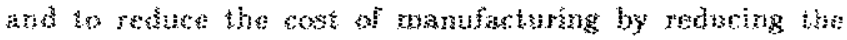

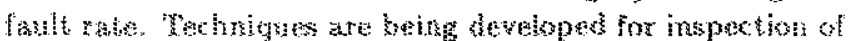

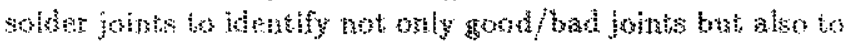

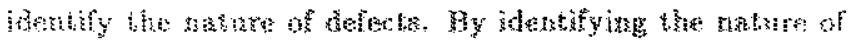

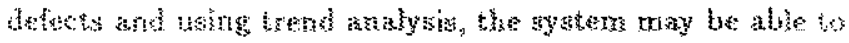

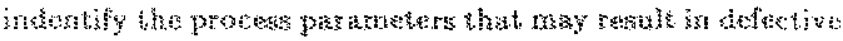

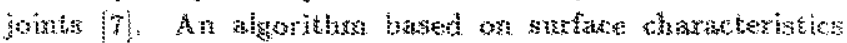

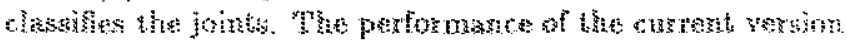

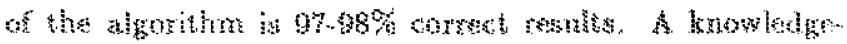

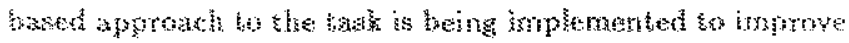

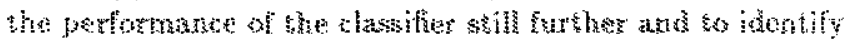

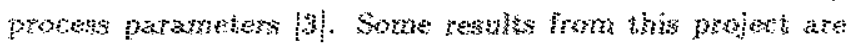

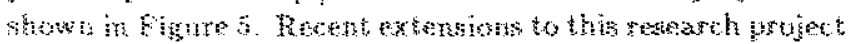

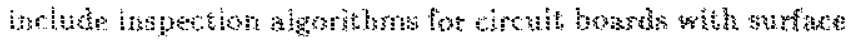
nontated componentat.

\section{Q.2 Semiconductor Wafer Inopection}

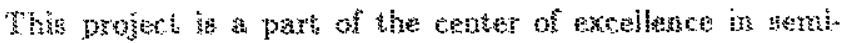

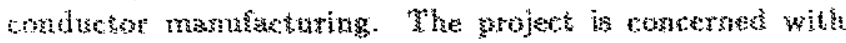

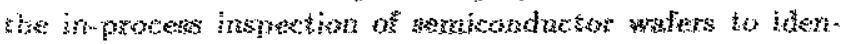

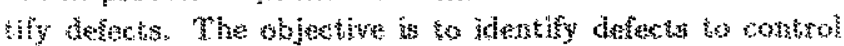

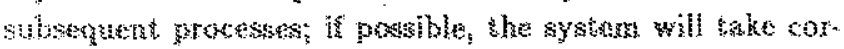

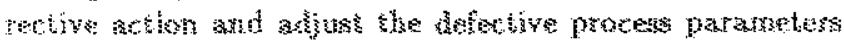

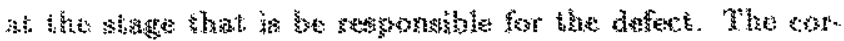

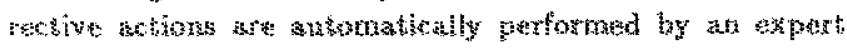

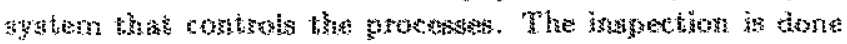

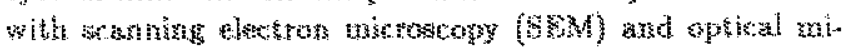

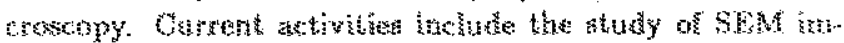

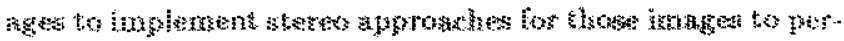

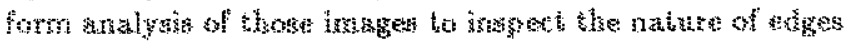

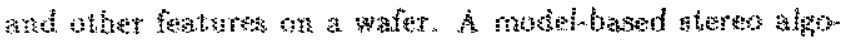

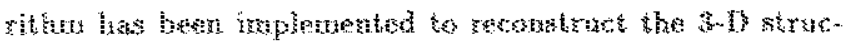

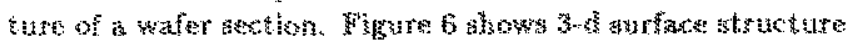

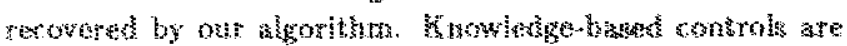
being deweloped for the intertace betwen the yinton syo wh and the oxpert systers in the antomated semicontactor mogufacturing protes:

\section{References}

II Agrawal, D, D, and D. Isin, "A piputhed potutopat

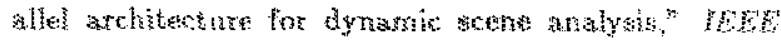

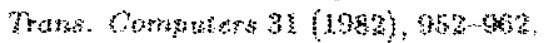

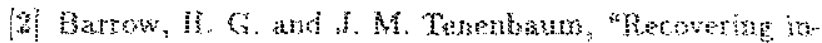

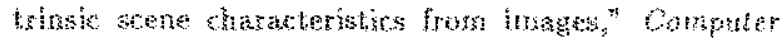

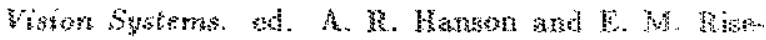

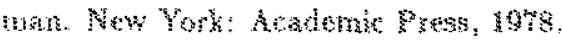

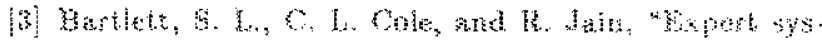

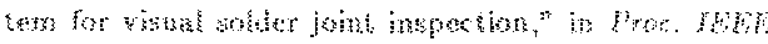

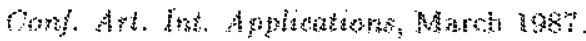

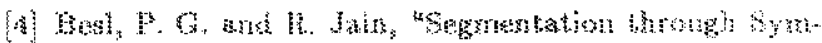

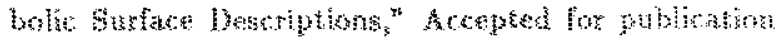

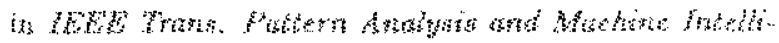
senter.

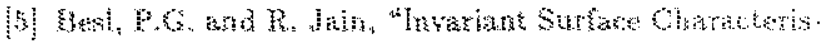

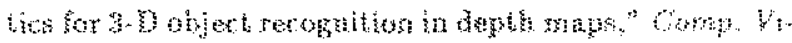

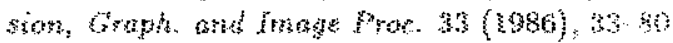

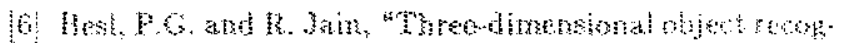

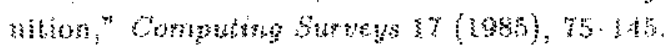

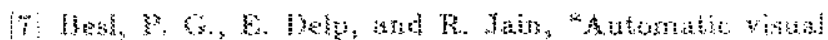

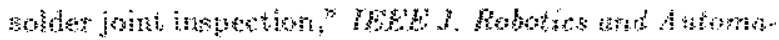
lions $(5069), 4256$.

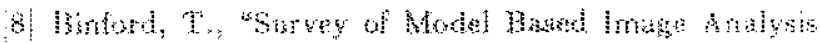

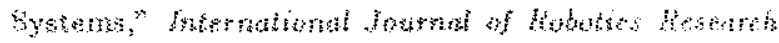
$1(1982), 18.64$

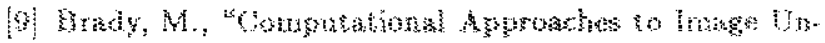

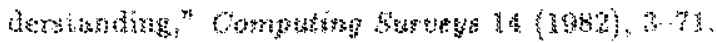

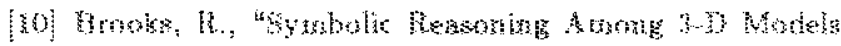

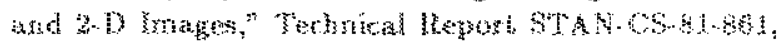

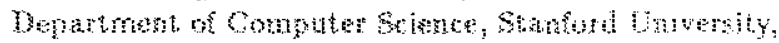
Stanford, Callornis, , hne $198 \mathrm{~s}$.

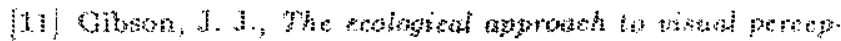
tho Boton Honghon Muffer, 1979.

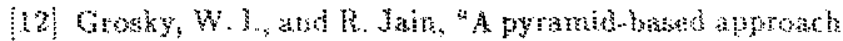
to segrentabn applied to regton mathing" Acopted

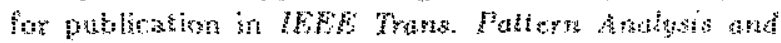
Machine folsiligenet

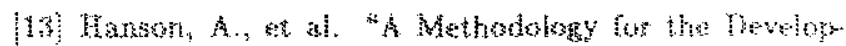

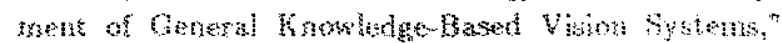

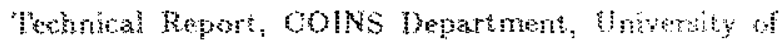
Maskehmets, Soptente: $\$ 985$.

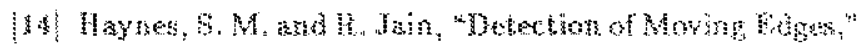

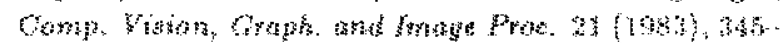
sent.

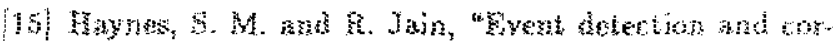

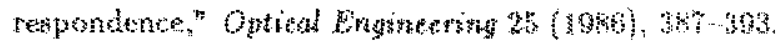

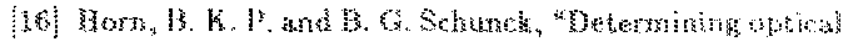

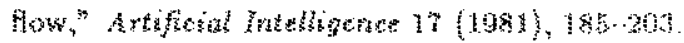


[17] Horn, B. K. P., Robot Vision. Cambridge: M. I. T. Press, 1986.

[18] Jain, R., Bartlett, S., and N. O'Brien, "Motion Stereo Using Egomotion Complex Logarithmic Mapping," Accepted for publication in IEEE Trans. Pattern Analysis and Machine Intelligence.

[19] Jain, R., D. Militzer, and H.-H. Nagel, (1977) "Separating non-stationary from stationary scene components in a sequence of real world TV-images," in Proc. Int. Joint Conf. Artificial Intelligence, pp. 612-618.

[20] Jain, R. and H.-H. Nagel, (1979) "On the analysis of accumulative difference pictures from image sequences of real world scenes," IEEE Trans. Patt. Anal. and Mach. Intel. 1 (1979), 206-214.

[21] Jain, R., "Direct computation of the focus of expansion," IEEE Trans. Patt. Anal. and Mach. Intel. 5 (1983), 58-64.

[22] Jain, R. "Segmentation of frame sequences obtained by a moving observer," IEEE Trans. Patt. Anal. and Mach. Intel. 6 (1984), 624-629.

(23) Jain, R. "Difference and Accumulative Difference Pictures in Dynamic Scene Analysis," Image and Vision Computing 2 (1984), 99-108.

[24] Jain, R., T. Sripradisvarakul, and N. O'Brien, "Symbolic Surface Descriptors for 3-D Object Recognition," in Proc. SPIE, January 1987.

[25] Jain, R. and N. O'Brien, "Ego-Motion Complex Logarithmic Mapping," in Proc. SPIE, November 1984.

[26] Jerian, C. P. and R. Jain, "Determining motion parameters for scenes with translation and rotation," in Proc. Workshop on Motion, Toronto, 1983.

[27] Kayaalp, A. E. and R. Jain, "A knowledge-based automatic on line wafer inspection system," in Proc. Vision 85, pp. $5.117-5.130,1985$.

[28] Knoll, T. F. and R. Jain, "Recognizing partially visible objects using feature indexed hypothesis, " IEEE J. Robotics and Automation 2 (1986), 3-13.

[29] Liou, S. P. and R. Jain, "Detecting Road Edges Using Hypothesized Vanishing Points," Accepted for publication in Comp. Vision, Graph. and Image Processing.

[30] Macworth, A., "Vision Research Strategy: Black Magic, Metaphors, Mechanisms, Miniworlds, and Maps," Computer Vision Systems. ed. A. Hanson and E. Riseman. New York: Academic Press, 1978.

[31) Neisser, U., Cognition and Reality. San Francisco: W. H. Freeman, 1976.

[32] O'Brien, N. G. and R. Jain, "Axial motion stereo," in Proc. Workshop on Computer Vision, Annapolis, Maryland, 1984.
[33] Rao, A. R., R. Jain, and T. E. Weymouth, "Knowledgebased vision systems," Submitted to Computing Surveys.

[34] Rao, A. R. and R. Jain, "Knowledge representation and control in computer vision systems," Accepted for publication in IEEE Expert.

[35] Schunck, B. G. "Motion segmentation and estimation," Doctoral Thesis, Department of Electrical Engineering and Computer Science, M. I. T., 1983.

[36] Schunck, B. G. "The motion constraint equation for optical flow," in Proc. Int. J. Conf. Pattern Recognition, pp. 20-22, Montreal, Canada, 1984.

[37) Schunck, B. G. "Motion segmentation and estimation by constraint line clustering," in Proc. Workshop on Computer Vision, pp. 58-62, Annapolis, Maryland, 1984.

[38] Schunck, B. G. "Surface-based smoothing of optical flow fields," in Proc. Conference on Intelligent Systems and Machines, pp. 107-111, Oakland University, Rochester, Michigan, 1984.

[39] Schunck, B. G. and B. K. P. Horn, "Constraints on optical flow computation," in Proc. Conf. Pattern Recog. nition and Image Processing, pp. 205-210, 1981.

[40] Schunck, B. G. "Image Flow: Fundamentals and Future Research," in Proc. Conf. Computer Vision and Pattern Recognition, San Francisco, 1985.

[41] Sethi, I.K. and R. Jain, "Finding trajectories of feature points in a monocular image sequence," IEEE Trans. Patt. Anal. and Mach. Intel. 9 (1987), 56-73.

[42] Sethi, I.K., "A fast algorithm for recognizing nearest neighbors, " IEEE Trans. Systems, Man, and Cybernetics 11 (1981), 245-248.

[43] Sethi, I.K. and R. Jain, "Determining three dimensional structure of rotating objects using a sequence of monocular views," Technical Report, Wayne State University, 1984.

[44] Shah, M. A. and R. Jain, "Detecting Time Varying Corners," Computer Vision, Graphics, and Image Processing 28 (1984), 345-355.

[45] Ullman, S. The Interpretation of Visual Motion. Cambridge: M. I. T. Press, 1979.

[46] Weymouth, T. E., J. S. Griffith, A. R. Hanson, E. M. Riseman, "Rule Based Strategies for Image Interpretation," in Proc. AAAI, pp. 429-432, August 1983.

[47] Weymouth, T. E., "Using Object Descriptions in a Schema Network for Machine Vision," Ph. D. Dissertation, University of Massachusetts, April 1986. 